\title{
ARTICLE
}

\section{Purification of Kraft cellulose in mild conditions using choline acetate based deep eutectic solvents.}

Received 00th January 20xx, Accepted 00th January 20xx DOI: $10.1039 / x 0 x \times 00000 x$

\author{
Greta Colombo Dugoni, ${ }^{[a] \S \uparrow}$ Andrea Mezzetta, ${ }^{[b] \S \uparrow}$ Lorenzo Guazzelli, ${ }^{[b]}$ Cinzia Chiappe, $\left.{ }^{[b]}\right]_{+}$ \\ Monica Ferro, ${ }^{*[a]}$ and Andrea Mele ${ }^{*[a, c]}$
}

Dedication: This paper is dedicated to the memory of Professor Cinzia Chiappe, beloved friend, inspiring colleague and excellent researcher.

\begin{abstract}
Three novel deep eutectic solvents (DESs) were prepared with choline acetate (ChOAc) as hydrogen bond acceptor (HBA) and three hydrogen bond donors (HBD): glycolic acid (GlyA), levulinic acid (LevA) and imidazole ( $(\mathrm{m})$. They showed different, albeit remarkable capability of dissolving lignin (from $35 \%$ to $100 \%$ ) and hemicellulose (from $40 \%$ to $50 \%$ ). The solubility was first tested on commercially available hemicellulose in neat DES and in DES aqueous solution. The high values of solubility observed (22-45 wt. \%) demonstrated the potential of DESs as hemicellulose solvents. A proof of concept test was carried out on Kraft cellulose: our systems provided selective solubilisation of lignin and hemicellulose leading to cellulose purification, as demonstrated by the lignocellulosic content before and after DES treatment.
\end{abstract}

\section{Introduction}

The importance of cellulose as a raw material with prominent environmentally friendly and biocompatible properties compared with petroleum-based products ${ }^{1}$ is constantly growing. The cellulose content of the total annual biomass productions is estimated in about $1.5 \times 10^{12}$ Tons. $^{2}$ It is thus considered an almost inexhaustible source of both raw material for environmentally friendly and biocompatible products and source of $\mathrm{C}$ atoms for organic chemistry alternative to fossil sources. ${ }^{3}$ Cellulose functionalization ${ }^{4}$ affords derivatives such as carboxymethylcellulose, cellulose acetate, nitrocellulose, used in a broad range of applications, from food to pharma, from textile to paint industries. The final quality of the cellulose-based materials very much depends on the purity of starting cellulose, its batch-to-batch variability, reactivity and efficient quality control throughout the whole production line. Moreover, the recovery of processing

\footnotetext{
a. Department of Chemistry, Materials and Chemical Engineering "G. Natta" Politecnico di Milano, Piazza Leonardo da Vinci, 32, 20133, Milano, Italy.

E-mail: monica.ferro@polimi.it, andrea.mele@polimi.it

b. Department of Pharmacy, University of Pisa, Via Bonanno 33, 56126 Pisa, Italy.

c. Istituto di Scienze e Tecnologie Chimiche (SCITEC-CNR), via A. Corti 12, 20133

Milano, Italy.

tDeceased in 2019.

$\S$ Greta Colombo Dugoni and Andrea Mezzetta equally contributed to this work

Electronic Supplementary Information (ESI) available: [details of any

supplementary information available should be included here]. See DOI: $10.1039 / x 0 x \times 00000 x$
}

chemicals, reagents, and the purification methods are still open issues: these difficulties are still hampering the production of cellulose-based materials as valid and ecofriendly alternative to fossil fuel-based products. To overcome all the problems, industrial processes require and use high purity cellulose.

The industrial production of cellulose is generally obtained by the Kraft process. The first step consists of cooking woodchips in an alkaline solution at elevated temperature and pressure to dissolve lignin and leave fibres composed of cellulose and hemicellulose intact. The cellulose obtained at this stage typically contains 15-25 wt. \% hemicelluloses and 5-10 wt. \% lignin, depending on the wood species. Kraft cellulose, commonly used in paper industries, is not a suitable starting material for the industrial production of cellulose derivatives. Industries usually use cotton linters cellulose or purified cellulose. ${ }^{5,6}$ There are some drawbacks associated to this choice: i) Cotton cellulose is more expensive than Kraft cellulose $(2.7 € / \mathrm{kg} \text { vs } 0.4 € / \mathrm{kg} \text {, price for } 1 \text { ton })^{7}$. ii) The cultivation of cotton is extremely demanding in terms of water consumption and use of pesticides compared to that of many varieties of trees, thus rising environmental issues. ${ }^{6,8}$ iii) The alternative to Kraft cellulose is the purified cellulose, also known as dissolving pulp. Purified cellulose is obtained by a processes based on the sulfite pulping ${ }^{9}$ or pre-hydrolysis Kraft pulping ${ }^{10,11}$. Both these chemical treatments work at harsh conditions (high temperature, high pressure, and reagents harmful to the environment). 
In this scenario, and in the perspective of more sustainable processes, a wide range of non-chemical treatments have been developed in the last few years based on mechanical extrusion, ${ }^{12}$ milling, ${ }^{13}$ microwaves, ${ }^{14,15}$ ultrasounds, ${ }^{16,17}$ steam explosion, ${ }^{18,19}$ and hot-water treatment. ${ }^{20}$ Additionally, several chemical treatments were proposed to break the binding between the lignin and the polysaccharides (cellulose and hemicellulose), to remove the hemicellulose fraction and to improve the cellulose reactivity towards chemical reagents and enzymes. ${ }^{21,22}$ The most used treatments are based on dilute acids ${ }^{23}$ or alkali, ${ }^{24}$ ammonia, ${ }^{25}$ ozone, ${ }^{25}$ mixture of solvents and acids (organosolvs) ${ }^{26}$ and ionic liquids ${ }^{27}$ but only a few of them can be used at industrial or pilot scale level. In particular, ionic liquids (ILs) showed high efficiency as reaction media for lignocellulosic waste pretreatment, ${ }^{28-32}$ and several studies highlighted their potential for improving cellulose digestibility $^{33-35}$ by lignin dissolution. ${ }^{36,37}$ Nevertheless, all the industrial applications of lignocellulosic biomasses pretreatment based on ILs suffer from the high costs of ILs, thus posing limitations for large-scale development of ILsbased processes. ${ }^{38-41}$ Additionally, toxicity and long-term recyclability can also represent severe issues. ${ }^{42}$

For all these reasons, the use of green solvents for the treatment of biomass remains the main challenge for biomass processing. Moreover, higher product yields must be balanced with solvent costs (supply, operations/separations, and disposal). A valid alternative to ILs is represented by Deep Eutectic Solvents (DESs) that received considerable attention in different research fields since $2004,{ }^{31}$ particularly in biomass processing. ${ }^{43-46}$ DESs are systems formed from an eutectic mixture of Lewis and Brönsted acids and bases, which can contain a variety of anionic and cationic species. ${ }^{43}$ DESs are considered a new class of ILs, characterized by high stability, low toxicity, ease of preparation, biocompatibility, biodegradability and sustainability. ${ }^{47,48}$ With respect to the synthesis of an organic ligand, the preparation of a DES is easy, does not require purification steps and gives quantitative yield. ${ }^{49}$ Several promising DESs for the solubilisation of lignocellulosic biopolymers are reported in the literature. ${ }^{45,50,51}$ Some types of DESs, especially those based on lactic acid, malic acid and oxalic acid, have been reported to extract more than $90 \%$ of lignin from many biomasses. ${ }^{52,53}$ In contrast, cellulose and hemicellulose are reported to be poorly soluble in DESs. ${ }^{49,51}$ In particular Ren et al. measured the solubility of cellulose in traditional DESs obtaining the order:

ChCl:imidazole (2.48 wt. \%) > ChCl:urea (1.45 wt. \%) > $\mathrm{ChCl}$ :ammonium thiocyanate $(0.83 \mathrm{wt} . \%) .{ }^{54}$ Unfortunately, a relationship between molecular composition of the biomass and the DES solvent properties is not fully understood. Morais et al. reported a pioneering work about the selective solubilisation of xylan in kraft cellulose but using a mixture of DES (ChCl:urea and ChCl:acetic acid) in $\mathrm{NaOH}$ solution. ${ }^{55}$ Therefore, the development of DESs capable of dissolving cellulose and hemicellulose or able to separate cellulose from hemicellulose remains an outstanding goal and the first fundamental step in the more general problem of separation and exploitation of cellulose, hemicellulose and lignin. In this scenario, we present an approach based on three novel DES formulations able to selectively solubilize hemicellulose in high yields, thus providing an easy and efficient way of cellulosehemicellulose separation and valorization. The solubility data showed are based on commercially available hemicellulose. The three novel, halogen-free DES systems here presented (Table 1 and Fig. 1) are based on choline acetate (ChOAc), which was already reported as a biocompatible alternative to traditional ILs for the treatment of lignocellulosic material. ${ }^{56-58}$

Table 1. DESs prepared for this study

\begin{tabular}{llcl}
\hline HBA & HBD & $\begin{array}{c}\text { Molar ratio } \\
\text { (HBA/HBD) }\end{array}$ & Abbreviation \\
\hline $\begin{array}{l}\text { Choline } \\
\text { acetate }\end{array}$ & Glycolic acid & $1: 1$ & ChOAc:GlyA \\
$\begin{array}{l}\text { Choline } \\
\text { acetate }\end{array}$ & Levulinic acid & $1: 1$ & ChOAc:LevA \\
$\begin{array}{l}\text { Choline } \\
\text { acetate }\end{array}$ & Imidazole & $1: 1$ & ChOAc:Im \\
\hline
\end{tabular}

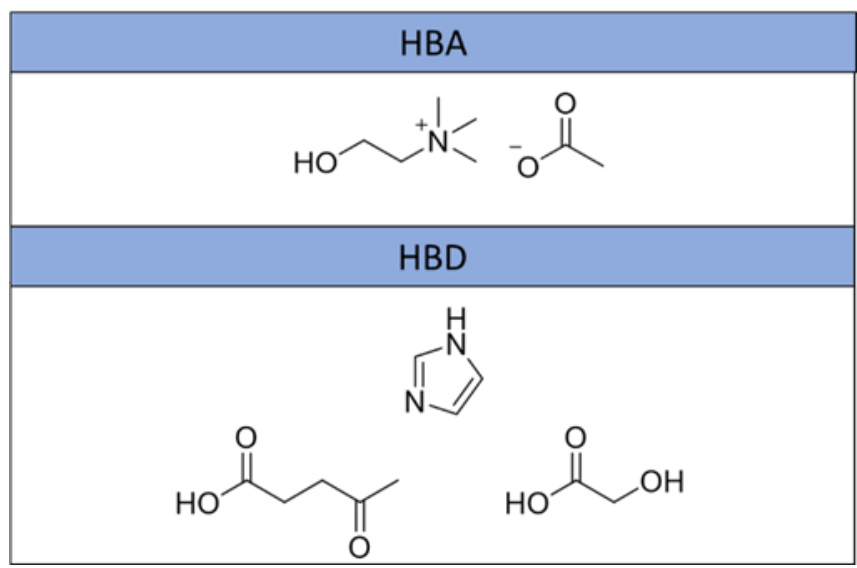

Fig. 1. Structure of HBA (Choline acetate) and HBDs (Levulinic acid, Imidazole and Glycolic acid) used to prepare DESs.

The addition of water to DES was also studied. ${ }^{59}$ Indeed, water plays a fundamental role in improving the extraction performance of DESs which are able to promote the dissolution of lignin. ${ }^{60}$ The role of water in modulating the physico-chemical properties of DES is still a hot topic of the fundamental and applied research. ${ }^{61,62}$ As remarked, ${ }^{62}$ the addition of water to DES may lead to unpredictable, but often benign, change of the DES properties. For these reasons, the novel DESs were tested for hemicellulose solubilisation in both neat and in the presence of different amounts of water. Finally, the performance of the proposed DESs as solvents in biomass treatment was tested on a Kraft cellulose containing hemicellulose and lignin. The recovery of purified cellulose 
with higher cellulose content due to removal of hemicellulose and lignin components is reported as a proof-of-concept. The paper is structured as follows: i) the characterization of the novel DES systems is presented and discussed, ii) the solvent properties towards reference hemicellulose are described and discussed, and iii) the case study of purification of Kraft cellulose by treatment with DES is reported.

\section{Results and discussion}

\section{ChOAc based DESs purity and viscosity}

The chemical purity of the three DESs was ascertained via ${ }^{1} \mathrm{H}$ and ${ }^{13} \mathrm{C}$ NMR spectroscopy in order to confirm the structure of the prepared DESs. The spectra are shown in Fig.S1-S6t. This analytical step is far from being unimportant, as it is reported $^{63}$, that DESs containing carboxylic acids as HBD may undergo esterification side-reaction with choline. In the present case, the ${ }^{1} \mathrm{H}$ NMR spectra of DESs after four weeks storage confirm that no esterification side-reactions occurred, thus providing important experimental evidence of the potential recyclability of DES in cellulose purification, as reported for choline chloride based DESs (Fig. S7†).

The three novel DESs were also characterized in terms of viscosity, thermal behaviour ${ }^{63}$ as well as thermal stability (See S8-15†).

The viscosity of DES depends on different parameters such as the chemical nature of the HBA and HBD, temperature and water content. ${ }^{43}$ DESs with low viscosity are desirable for the biomass processing and for the industrial point of view. ${ }^{50}$

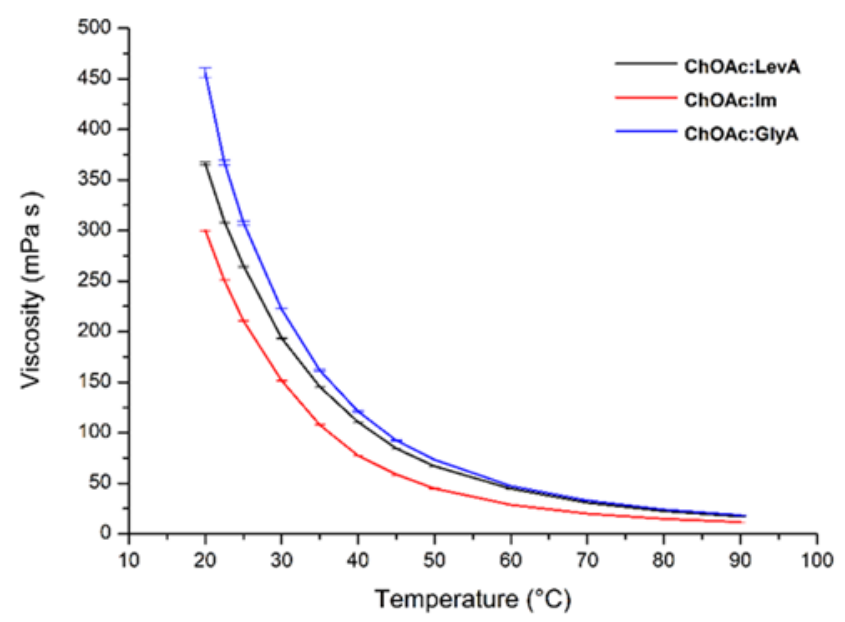

Fig. 2. Comparison of the viscosity behavior of ChOAc:LevA (black), ChOAc:Im (red), and ChOAc:GlyA (blue) as a function of the temperature.

As generally observed for DESs, the viscosity rapidly decreases on increasing the temperature (Fig. 2). As the HBA is the same and the water amount is similar (close to $200 \mathrm{ppm}$ ) for all DESs studied, the different viscosity values observed at $20^{\circ} \mathrm{C}$ (Table
$\left.\mathrm{S} 1{ }^{+}\right)$depend mostly on the HBDs' nature. In particular, imidazole-based DES is characterized by a lower viscosity than the corresponding glycolic and levulinic acid containing DESs. In the examined temperature range, the temperature dependence of viscosity follows, with good approximation, the Arrhenius equation (eqn 1 ) which can be defined as following:

$$
\ln \eta=\ln \eta_{\infty}+\frac{E_{a}}{R T} \quad[\text { eqn } 1]
$$

In this equation, $\eta$ is the dynamic viscosity ( $m P a s), \eta_{\infty}$ is the viscosity at infinite temperature ( $\mathrm{mPa} s$ ), and $E_{a}$ is the activation energy $(\mathrm{KJ} / \mathrm{mol})$ for viscous flows. Aparicio et al. ${ }^{64}$ reported that $E_{a}$, which describes the difficulty for particles to move, is correlated to the nature of HBD and HBA constituents. As mentioned above, the HBA (ChOAc) is the same for all DESs, hence the difference in activation energy is due to the different interactions with each HBD. Therefore, by comparing the activation energies $E_{a}$ of different DESs (Table $\left.\mathrm{S}^{+}\right)$, it is possible to gain an insight in the strength of hydrogen bond interactions of the HBA and the different HBDs, which is a crucial aspect when DESs are used in biopolymer dissolution. A comparison of the viscosities at $20^{\circ} \mathrm{C}$ of DESs studied in this work, and those featuring the most common $\mathrm{ChCl}$ as HBA and levulinic acid, glycolic acid and imidazole as HBD, was particularly interesting. $\mathrm{ChCl}: \mathrm{Im}$ is solid at room temperature (melting point $\left.54-56^{\circ} \mathrm{C}\right)^{65}$, hence the viscosity cannot be evaluated. For ChCl:LevA, the viscosity value of $320.3 \mathrm{cP}$ has been reported at higher molar ratio (1:2) and it is only slightly lower than $366.0 \mathrm{CP}$ observed for ChOAc:LevA, albeit at 1:1 molar ratio. ${ }^{66}$ Finally, ChOAc:GlyA DES displays a significantly lower viscosity (456.1 cP) than its counterpart ChCl:GlyA (779.4 CP). ${ }^{66}$

\section{Hemicellulose solubilisation: a model study}

In this work, three different DESs based on ChOAc and different hydrogen bonds donors (HBDs) were tested. HBDs were chosen on the basis of some structural features likely to modulate the interaction of the HBD with the xylan portion. A first DES was prepared with glycolic acid (GlyA), an $\alpha$-hydroxy acid with one carboxyl group and one hydroxyl group capable of forming hydrogen bonds. A second DES used levulinic acid (LevA), having one carboxyl and one carbonyl group and, finally, a third DES was prepared by using imidazole (Im). Last but not least, ChOAc:Im showed lower viscosity that the other two.

The solubility tests were carried out on a sample of hemicellulose commercially available. The sample was characterized by NMR in order to confirm the structure of 4-Omethyl glucuronoxylan (Fig. S20-S21+) and then used to study the solubility in ChOAc based DESs presented in the Introduction. The mixture of DES, prepared according to the experimental procedure (see Experimental Section), and 
hemicellulose was stirred at the fixed temperature of $80^{\circ} \mathrm{C}$. The hemicellulose was added by consecutive additions of $1 \mathrm{wt}$. $\%$ to the DES until saturation was reached. The DESs solutions containing hemicellulose were very viscous and with the brownish colour from the commercial hemicellulose. The amounts of soluble hemicellulose was evaluated by using the cloud point method. ${ }^{67}$ When the turbidity or the presence of particles was noticeable, the samples were equilibrated to check the disappearance of the cloudiness. When solution saturation was reached, a $1 \mathrm{wt}$. \% of water was added to the solution. In this way, it was possible to construct solubility graphs as a function of the percentage of water added in DES, up to $15 \%$ in weight. It is worth mentioning that even in the presence of such a large amount of water, we can assume that the DESs structure, characterized by a large and complex hydrogen bonding network, can be retained, and water participating as both HBD and HBA.$^{68,69}$

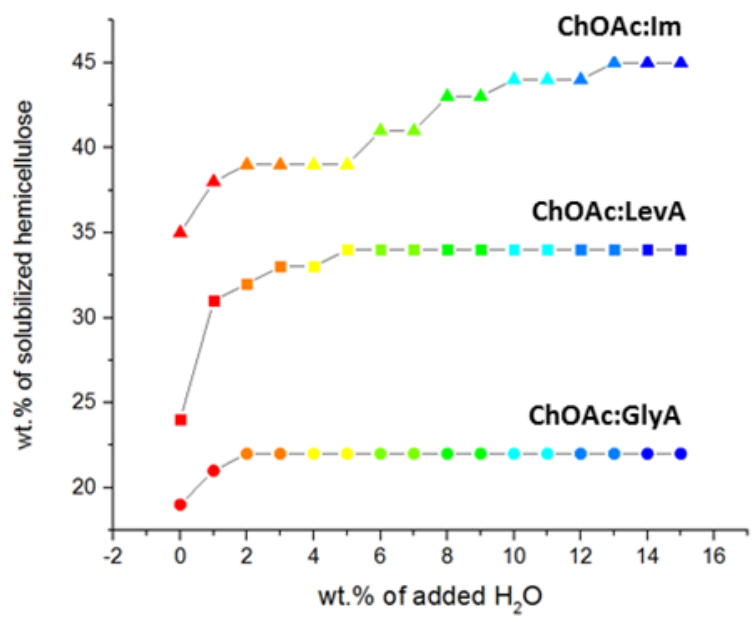

Fig. 3. Amount of soluble hemicellulose (wt.\%) as a function of added water content (wt.\%).

We underline that our protocol allowed us to test the hemicellulose solubility in both pure DES and water-in-DES systems. In the latter cases, additional amounts of hemicellulose were added to the water-in-DES solutions till the cloud point. ${ }^{70}$ The solubility data in this way achieved are of particular interest in view of industrial scale applications. Indeed, the presence of water without collapse of performance may represent a strong economic factor in favour of the DES based process for biomass fractionation and valorization. The experimental points of the curves reported in Fig. 3 thus show the hemicellulose solubility in mixed DESwater systems as a function of the water content. The steps described above were repeated up to the maximum hemicellulose wt. \% experimentally detectable. The curves show that the worst performing DES is ChOAc:GlyA. The solubility of hemicellulose, in this case, is $19 \%$ in weight with respect to the solvent. The addition of water leads to a modest increase of solubility up to $22 \mathrm{wt}$. \%. The ChOAc:LevA DES displayed a larger solubilizing power towards hemicellulose when water was added. Indeed, 24 wt. \% hemicellulose is dissolved in the pure DES, whilst $31 \mathrm{wt}$. \% and $34 \mathrm{wt}$ \% dissolution are reached after the addition of $1 \mathrm{wt}$. \% and $5 \mathrm{wt}$. $\%$ of water, respectively. However, further addition of water did not improve the solubility. The ChOAc:Im DES was the best performing system, providing $35 \mathrm{wt}$. \% of dissolved hemicellulose without water addition. A significant improvement of hemicellulose solubility was observed upon progressive addition of water, without the plateau of the other two samples. The maximum solubility value within the explored DES-water composition range was $45 \mathrm{wt}$. \% of hemicellulose dissolved in 15 wt. \% water containing DES. Finally, the experimental points of Fig. 3 reveal two different behaviors: the ChOAc:GlyA and ChOAc:LevA on one side, and the ChOAc:Im on the other. The first two systems share the initial hemicellulose solubility burst followed by a plateau. The ChOAc:Im, on the contrary, shows constantly increasing solubility enhancement with added water.

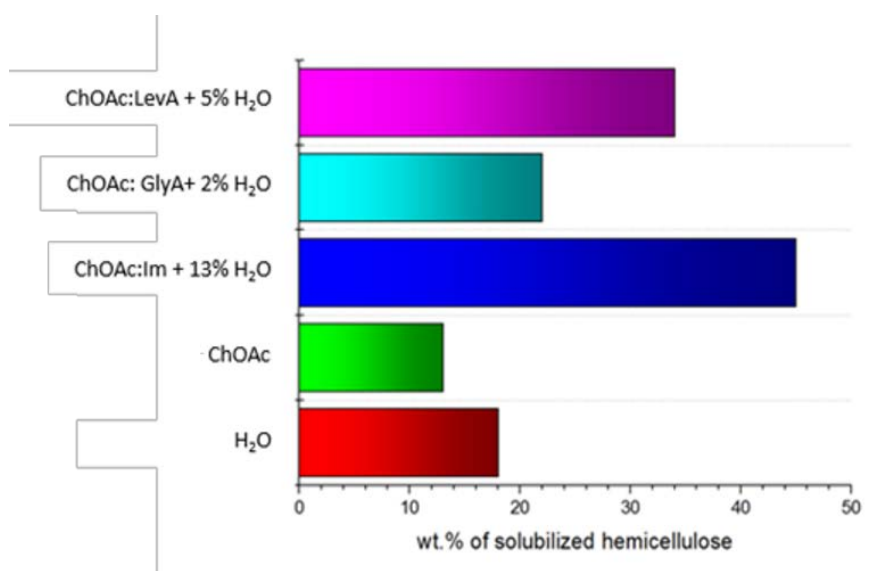

Fig. 4. Comparison of maximum values of hemicellulose solubility obtained in the novel choline acetate-based DESs +wt.\% water, choline acetate IL and water.

The solubility values of hemicellulose in DES herein described can be compared with the corresponding values reported in the ionic liquid choline acetate ${ }^{58}$ and the solubilization in water at the same temperature $\left(80^{\circ} \mathrm{C}\right.$, this work $): 13 \mathrm{wt} . \%$ and $18 \mathrm{wt}$. $\%$, respectively. The results, summarized in Fig.4, point out the better performance of DESs compared to ILs and the synergic effect of DES and water on hemicellulose solubilisation.

To better understand the role of choline acetate, solubility tests on hemicellulose using choline chloride $(\mathrm{ChCl})$ and betaine (Bet) as HBA were performed. The results are shown in Table 2. Entries 3, 6 and 9 refer to the choline acetate-based DESs, the other entries to the homologous DESs obtained from traditional choline chloride and betaine. The tests were carried out on the pure DESs and on the mixture with $15 \mathrm{wt} \%$ water. The results can be summarized as follows: i) The direct comparison of the results for ChCl:LevA vs ChOAc:LevA (entry 
1 vs 3 ) and ChCl:Gly vs ChOAc:Gly (entry 4 vs 6 ) indicate the superior performance of the acetate-based DES for hemicellulose dissolution both in the pure systems and in the water containing systems (fourth and fifth column, respectively). Same trend is observed when comparing betaine-based DES with choline acetate-based DES (entry 2 vs 3 and 5 vs 6). ii) The entries related to DESs containing imidazole deserve further comments. The anhydrous systems (fourth column, entries 7, 8 and 9) confirm the picture described above. The data of the fifth column show the unexpected and intristing synergic action of water and imidazole for all the systems (fifth column, entries 7, 8 and 9) Nevertheless, the ChOAc:Im DES outperformed the two traditional DESs (45 wt\% hemicellulose solubilization vs 18 wt $\%$ and 5 wt\% of ChCl:Im and Bet:Im, respectively). Overall, the results of Table 1 show that choline acetate-based DESs are promising media for hemicellulose solubilization with higher performance compared to traditional choline chloride or betaine analogues. From the structural standpoint, the novel finding of a synergistic action of imidazole and water prompts for deeper investigation of the complex network of interaction taking place in DESs in that range of water content.

Table 2. Hemicellulose solubility results for different DESs studied. Reference systems based on choline chloride and on betaine are also included for comparison.

\begin{tabular}{ccccc}
\hline Entry & HBA & HBD & $\begin{array}{c}\text { Solubility } \\
\text { in DES } \\
\text { (wt. \%) }\end{array}$ & $\begin{array}{c}\text { Solubility in DES } \\
+15 \mathbf{H}_{\mathbf{2}} \text { O wt. \% } \\
\text { (wt. \%) }\end{array}$ \\
\hline $\mathbf{1}$ & ChCl & LevA & $<1$ & $<1$ \\
\hline $\mathbf{2}$ & Bet & LevA & $<1$ & $<1$ \\
\hline $\mathbf{3}$ & ChOAc & LevA & $\mathbf{2 4}$ & $\mathbf{3 4}$ \\
\hline $\mathbf{4}$ & ChCl & GlyA & $<1$ & $<1$ \\
\hline $\mathbf{5}$ & Bet & GlyA & $<1$ & $<1$ \\
\hline $\mathbf{6}$ & ChOAc & GlyA & $\mathbf{1 9}$ & $\mathbf{2 2}$ \\
\hline $\mathbf{7}$ & ChCl & Im & $<1$ & 18 \\
\hline $\mathbf{8}$ & Bet & Im & $<1$ & 5 \\
\hline $\mathbf{9}$ & ChOAc & Im & $\mathbf{3 5}$ & $\mathbf{4 5}$ \\
\hline
\end{tabular}

Finally, a simple test on cellulose solubilization was also carried out with microcrystalline cellulose (MCC). The mixture of DES and MCC was stirred at the constant temperature of $80{ }^{\circ} \mathrm{C}$. Test with wt. $1 \%$ MCC in all of the three DESs did not show any appreciable dissolution of MCC. The three DESs here described are thus not suitable for the solubilisation of cellulose.

Hemicellulose fraction was then recovered from the solution by adding a mixture of water and ethanol 1:5 v/v. In order to remove the residual DES, the precipitate was washed 4 times with ethanol and treated in batches with the cation exchange resin Amberlite IR-120. The recovered hemicellulose (85-92\% in weight) was then analyzed by NMR and compared with the pristine hemicellulose (Fig. S22 ${ }^{\dagger}$ ). The ${ }^{1} \mathrm{H}$ NMR indicates that the recovery protocol is efficient, providing recovered hemicellulose with no significant structural modification with respect to the pristine hemicellulose.

\section{Proof of concept: Kraft cellulose treatment}

Pure cellulose, named also dissolving grade pulp, with low content of hemicellulose, lignin and minerals, is widely used for the production of cellulose derivatives, especially for cellulose acetate or textile fibres. ${ }^{71}$ However, the high costs related to the production of this pulp has raised interest in the production of dissolving cellulose from paper grade cellulose through the selective removal of hemicellulose. Herein a purification process of Kraft cellulose using green solvents and mild conditions is proposed. For this purpose, a paper grade Kraft cellulose with a high content of hemicellulose (26 wt.\%) and $6 \mathrm{wt}$. \% lignin content (Table 2) was chosen to validate the DESs treatment. The structure of hemicellulose present in the Kraft cellulose was first characterized by NMR after alkaline treatment. In this way the structure of the hemicellulose present in the Kraft cellulose was assessed and then compared to the hemicellulose obtained after both the alkaline and the DESs treatments. To this end, a sample of Kraft cellulose (1g) was treated with $\mathrm{NaOH} 18 \mathrm{wt}$. \% overnight at $25^{\circ} \mathrm{C}$. The solution was then neutralized with Amberlite IR-120 resin, dried and re-suspended in $D_{2} O$. The hemicellulose fraction was characterized by the 2D NMR experiments HSQC and TOCSY (Fig. S24-S25 ${ }^{\dagger}$ ). The peaks assignment, also supported by literature data, ${ }^{72,73}$ highlighted the presence of Xylose $(X)$, Glucose (Glc) and Mannose (Man) units as major components, and of Galactose (Gal) and Arabinose (A) as minor components. The observed signals are compatible with galactoglucomannans and arabinoglucuronoxylans, which are the primary and secondary components in softwood. ${ }^{74}$ No glucuronic acid signals were detected, probably because of the low concentration, below the detection limit of the technique. The DES treatment effects on Kraft cellulose was evaluated in terms of: i) composition according to ISO 692:1982 standard (see Experimental for details), ii) physical chemical properties (crystallinity index, thermal behaviour and IR spectra), iii) technical benchmarks for cellulose: Fock's reactivity, intrinsic viscosity and R18.

The quantitative analysis of the lignocellulosic content of Kraft cellulose and cellulose after DESs treatment was carried out according to the ISO 692:1982 method. The results are described in Table 3. The result show that the DES treatment leads to the decrease of both lignin and hemicellulose content and the increase of cellulose from $67.7 \%$ (Kraft cellulose) to values in the $82.2-86 \%$ range. The initial lignin content was around $6.3 \%$. It was reduced to $4.1 \%$ and $2.1 \%$ after ChOAc:Im and ChOAc:GlyA, respectively. Interestingly, the lignin was completely removed after the treatment with ChOAc:LevA. The decrease of the hemicellulose content was in the $40-50 \%$ range.

Table 3. Yield of recovered cellulose after DES treatment (cellulose recovery \%), quantification of lignocellulosic content (cellulose composition) of the enriched cellulose fractions according to ISO 692:1982 standard for all studied samples and 
crystallinity index (C.I.\%) of cellulose and cellulose treated with DESs evaluated by $\mathrm{XRD}$. The asterisk* indicates cellulose II.

\begin{tabular}{cccccc} 
Sample & $\begin{array}{c}\text { Cellulose } \\
\text { recovery } \%\end{array}$ & \multicolumn{3}{c}{ Cellulose composition } & C.1.\% \\
\cline { 3 - 6 } & & Cellulose \% & Lignin \% & Hemicellulose \% & \\
\hline Kraft Cellulose & - & $67.7 \pm 0.1$ & $6.3 \pm 1.1$ & $26 \pm 0.2$ & $34 \pm 2$ \\
\hline $\begin{array}{c}\text { Cellulose treated } \\
\text { with } \mathrm{NaOH}\end{array}$ & $82.7 \pm 1.2$ & $89.7 \pm 0.8^{*}$ & $3.8 \pm 0.5$ & $6.5 \pm 0.9$ & Cellulose II \\
\hline $\begin{array}{l}\text { Cellulose treated } \\
\text { with ChoAc:GlyA }\end{array}$ & $88.5 \pm 0.8$ & $82.2 \pm 1.4$ & $2.1 \pm 0.4$ & $15.7 \pm 1.6$ & $54 \pm 3$ \\
\hline $\begin{array}{l}\text { Cellulose treated } \\
\text { with ChoAcilm }\end{array}$ & $84.3 \pm 1.4$ & $82.8 \pm 0.8$ & $4.1 \pm 0.5$ & $13.1 \pm 0.8$ & $53 \pm 3$ \\
\hline $\begin{array}{c}\text { Cellulose treated } \\
\text { with ChOAcilevA }\end{array}$ & $86.7 \pm 1.8$ & $86.0 \pm 1.2$ & $0 \pm 0.2$ & $14.0 \pm 0.4$ & $54 \pm 3$ \\
\hline $\begin{array}{l}\text { Cellulose treated } \\
\text { with ChOAcilevA } \\
+15 \text { wt. \% } \mathrm{H}_{2} \mathrm{O}\end{array}$ & $88.4 \pm 0.4$ & $85.5 \pm 0.5$ & $0 \pm 0.5$ & $14.5 \pm 0.4$ & $52 \pm 3$ \\
\hline
\end{tabular}

In Table 3 a cold caustic treatment of cellulose is reported as comparison with the DES treatment. For the correct data interpretation, it is important to underline that the conversion of cellulose I polymorph (native cellulose) into cellulose II occurs after caustic treatment only. ${ }^{75}$

The best result was obtained by using ChOAc:Im. Such a system successfully removed $50 \%$ of hemicellulose. A further test was performed using DES ChOAc:LevA containing $15 \mathrm{wt}$. $\%$ water. Noteworthy, neat ChOAc:LevA and the same DES in the presence of $15 \mathrm{wt}$. \% water showed comparable results: these data reveal that such a DES retains its activity even with quite a high water content. This result could have interesting implications from an industrial point of view. Indeed, the beneficial effect of water on the viscosity of these media is well-known and this would consequently reduce the operational costs of the process. Furthermore, in the recycling step, a substantial reduction of energy costs (more than 3500 $\mathrm{KJ}$ per $\mathrm{Kg}$ of Kraft cellulose treated) can be envisaged by avoiding the water removal.

Kraft cellulose samples, after DES treatments, were analysed by $X R D, T G A$ and IR to understand if significant changes in the structure ensued. The Powder XRD patterns of Kraft cellulose and recovered cellulose after DES treatment are reported in

Fig.S27-32 $\dagger$. The diffractograms show some common features: all the samples present broad peaks at $2 \theta 14.7^{\circ}, 16.8^{\circ}, 22.7^{\circ}$ and $34.8^{\circ}$, corresponding to the reflection planes (1-10), (110), (200), (004), respectively, consistent with the cellulose I crystal lattice. ${ }^{64,76}$ The X-ray data show that cellulose I lattice is preserved upon treatment in DES. Indeed, no reflexes assignable to cellulose II are present in the diffractograms of cellulose treated with DESs. The intensity differences around $2 \theta=20^{\circ}$ indicate a decrease of the amorphous halo (Fig. $\left.\mathrm{S} 28^{\dagger}\right) .{ }^{77}$ Given that amorphous regions derive from different components in lignocellulosic materials (e.g. cellulose amorphous regions, lignin, hemicellulose), the increase of apparent crystallinity of the cellulose showed by the increase of the crystallinity index actually represents an indirect proof of the successful purification treatment. ${ }^{78}$ Therefore, the crystallinity index (C.I. \%) was evaluated of each sample (Table 3). It was assessed as the ratio between the area of the crystalline peaks $\left(A_{C r}\right)$ and the total area of the spectrum (crystalline peaks and amorphous halo) $\left(\mathrm{A}_{\text {TOT }}\right.$ ) (eqn 2). The peaks areas were evaluated by peaks deconvolution (Fig. S28S32†).

$$
\text { C.I.\% }=\frac{A_{C r}}{A_{T O T}} \cdot 100 \quad[\text { eqn } 2]
$$

The C.I. \% values (Table 3) confirm the increase of the crystallinity for all the samples treated with DES, including that containing $15 \mathrm{wt}$ \% water. This suggests a reduction of the amorphous content after the DES treatment as already demonstrated in the work of Pan et al. ${ }^{49}$ This means that the tested DESs efficiently dissolve the amorphous components present in Kraft cellulose (hemicellulose and lignin) which contribute to the amorphous halo in Powder XRD diffractograms of cellulose. Cellulose samples treated with DES show similar crystallinity, in the range $52-54 \%$ (Table 3 ) and markedly higher that the starting Kraft cellulose (KC, 34\%). This is coherent with the decrease of the amorphous components from ca. $29 \%(\mathrm{KC})$ to values in the $14-17 \%$ range (DES treated cellulose). The C.I.\% of cellulose sample treated with $\mathrm{NaOH}$ cannot be compared with the others C.I. \% values because, after this treatment, cellulose $\mathrm{I}$ is converted in the thermodynamically more stable polymorph cellulose II.

In considering the consistency of the data, it should be kept in mind that the uncertainty associated to $\mathrm{Cl} \%$ can reach values as high as \pm 6 , due to the sum of the instrumental measurement error and to the deconvolution of the peaks, as previously reported in the literature. ${ }^{79}$

A comparison of the thermal stabilities, determined by TGA, of pristine Kraft cellulose with those obtained after the DESs treatment (Fig. 5, Fig. S33-S34†) corroborates the finding of the XRD analysis. Indeed, higher decomposition temperatures than the pristine Kraft cellulose were observed for the regenerated celluloses, which can be ascribed to the apparent higher crystallinity of cellulose and the removal of amorphous material. 


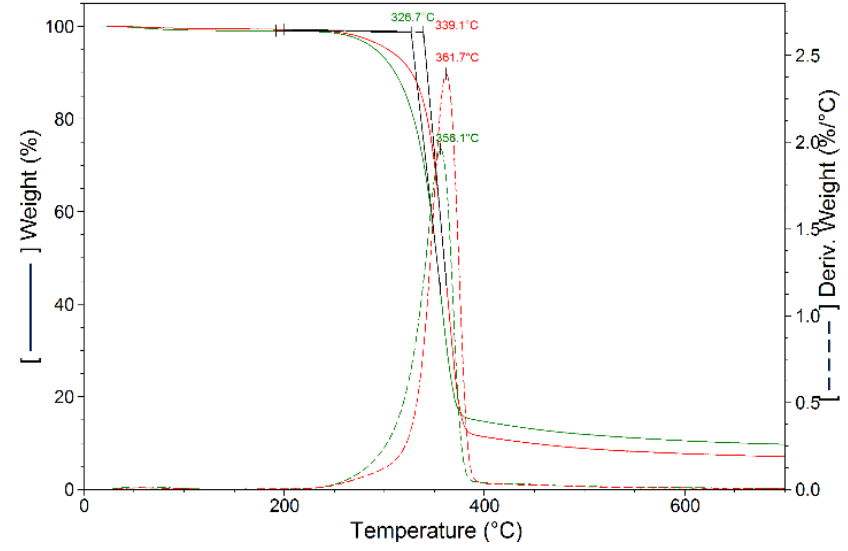

Fig. 5. Comparison of TG and DTG curves of dissolved and regenerated Kraft cellulose (KC, red line) and pristine KC (green line).

FT-IR spectra of cellulose and cellulose after DESs treatment are reported in Fig. S35-S39+. The sample were analysed with the same concentration in $\mathrm{KBr}$ and the same thickness of pellets. It is possible to observe the presence of differences among the intensities of some absorption bands. In detail, there are some differences between Kraft cellulose and cellulose treated with DES in the band at $1465 \mathrm{~cm}^{-1}$ due to the asymmetric deformation of $\mathrm{C}-\mathrm{H}$ bonds: this finding is considered as a marker of the presence of lignin. ${ }^{80}$ In addition, differences are observed in the band at $1044 \mathrm{~cm}^{-1}$, which is uniquely assigned to $\mathrm{C}-\mathrm{O}, \mathrm{C}-\mathrm{C}$ stretching or $\mathrm{C}-\mathrm{OH}$ bending in hemicellulose. These data are consistent with a lower amount of lignin and hemicellulose in the sample treated by DESs.

Finally, the technical characteristics of the enriched cellulose fractions were assessed by using the following parameters: i) Fock's reactivity, ii) intrinsic viscosity and iii) R18 (see
Experimental for definition and use). The results are shown in Table 4.

Reactivity is often the most significant quality parameter of dissolving pulps. ${ }^{81,82}$ Using cellulose with a low hemicellulose content improves the quality of cellulose derivatives and reduce the demands of reagents, production costs and the environmental impact. ${ }^{82}$ The reactivity of the treated cellulose was analyzed according to a modified version of Fock's method. ${ }^{83,84}$ The reactivity values, expressed as regenerated cellulose yield, are reported in Table 4.

Table 4. Fock's reactivity, intrinsic viscosity and R18 of cellulose obtained after DES treatment.

\begin{tabular}{cccc}
\hline Sample & $\begin{array}{c}\text { Fock's Reactivity } \\
\text { (\%) }\end{array}$ & $\begin{array}{c}\text { Intrinsic Viscosity } \\
\text { (ml/g) }\end{array}$ & R18 \\
\hline $\begin{array}{c}\text { Cellulose treated } \\
\text { with ChOAc:GlyA }\end{array}$ & 88.2 & 869 & 84.6 \\
\hline $\begin{array}{l}\text { Cellulose treated } \\
\text { with ChOAc:Im }\end{array}$ & 69.6 & 994 & 87.4 \\
\hline $\begin{array}{l}\text { Cellulose treated } \\
\text { with ChOAc:LevA }\end{array}$ & 79.70 & 876 & 86.0 \\
\hline
\end{tabular}

The Fock's reactivity values are in the expected range for dissolving grade cellulose. ${ }^{82,85}$

The viscosity of the treated pulps determined as the intrinsic viscosity of a sample of cellulose dissolved in a cuproethylenediamine hydroxide solution (0.5M) was calculated according to ASTM D1795 with Cannon Fenske Ubbelohde capillary viscosimeter. Viscosity measurements

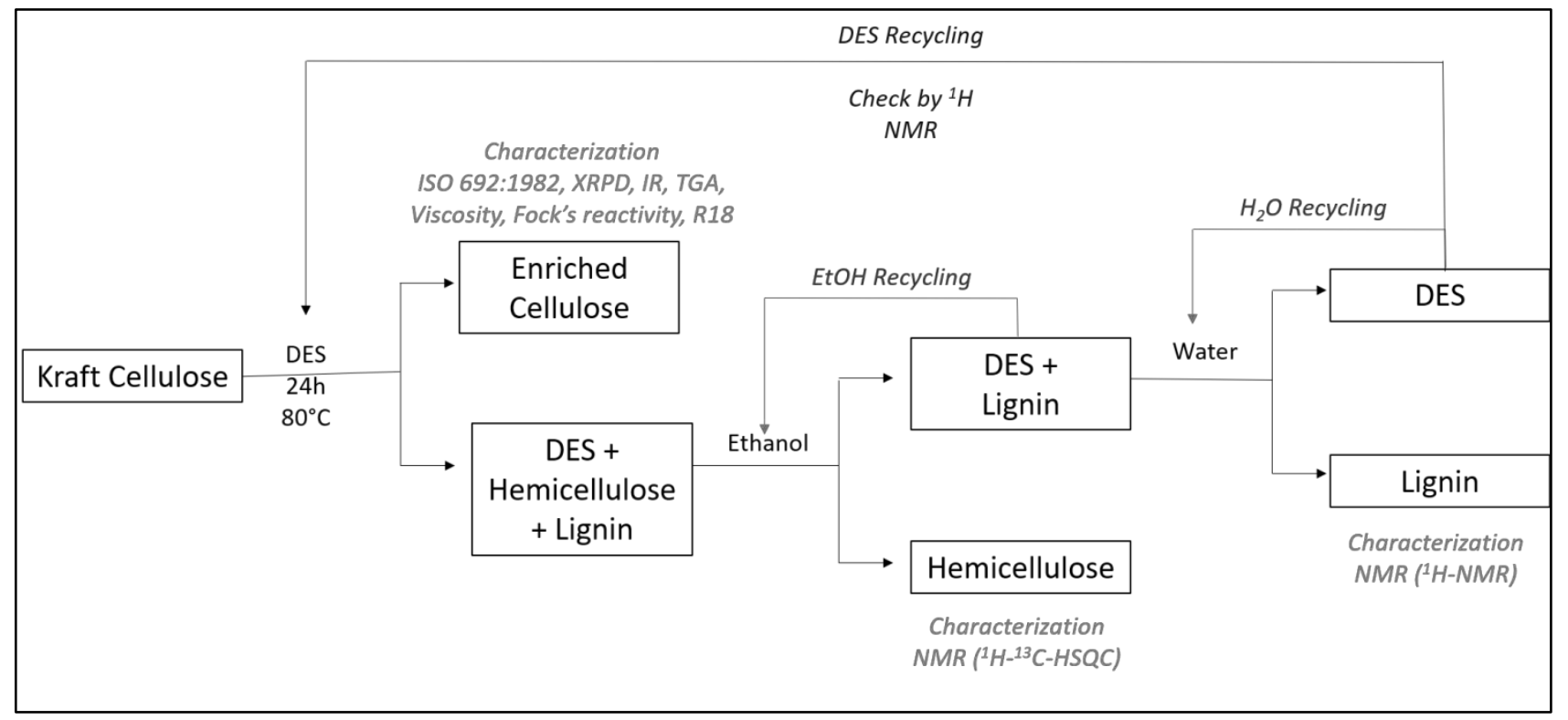

Scheme 1. Simplified process flow diagram for Kraft cellulose treatment using ChOAc-based DES 
were carried out in duplicate and the results were reported in Table 4. Once again, the typical intrinsic viscosity values of commercial dissolving grade cellulose are comparable with those of the enriched cellulose samples of Table $4^{85}$.

As a third descriptor we report the measured R18 values. Basically, R18 accounts for the solid residue without hemicellulose. It is a common benchmark for dissolving grade cellulose. In this case too, the R18 values fall in the range for dissolving grade cellulose. ${ }^{86}$

Overall, the Kraft cellulose purification process proposed in this work for the PoC can be sketched as in Scheme 1. The scheme summarizes the fractionation pathway, the recovery steps, the characterization methods and the recycling route of the solvents. The remaining part of this section describes the characterization of the dissolved fractions. The hemicellulose fraction was analysed by NMR spectroscopy using ${ }^{1} \mathrm{H}$ spectrum (Fig. S24+) and HSQC (Fig. S25') experiments. As mentioned above, the spectra were compared with the spectra of hemicellulose achieved by alkaline treatment. ${ }^{1} \mathrm{H}$ NMR spectra (Fig. S24t) showed that a significant fraction of lignin was present in the hemicellulose from alkaline treatment. In contrast, no traces of lignin were detected in the hemicellulose obtained from DES treatment. This suggests that the DES treatment proposed in this work provides an efficient separation of hemicellulose from cellulose and lignin, and the hemicellulose recovery in a clean way. As shown in Scheme 1, the simple addition of water to DES solution permits to recover by filtration the lignin component and recycle the solvent. The ${ }^{1} \mathrm{H}$ NMR spectrum of lignin dissolved in d6-DMSO is also reported in Fig S26+. The spectrum also clearly shows the absence of residual DES.

The HSQC spectrum of the recovered hemicellulose recorded in $\mathrm{D}_{2} \mathrm{O}$ is reported in Fig. 6 . The corresponding $\mathrm{HSOC}$ spectrum of the hemicellulose obtained after the alkaline process is shown in Fig. S25. The comparison of the two HSQC plots clearly indicates that during the DES treatment there is no preferential solubilization of any hemicellulose fractions.

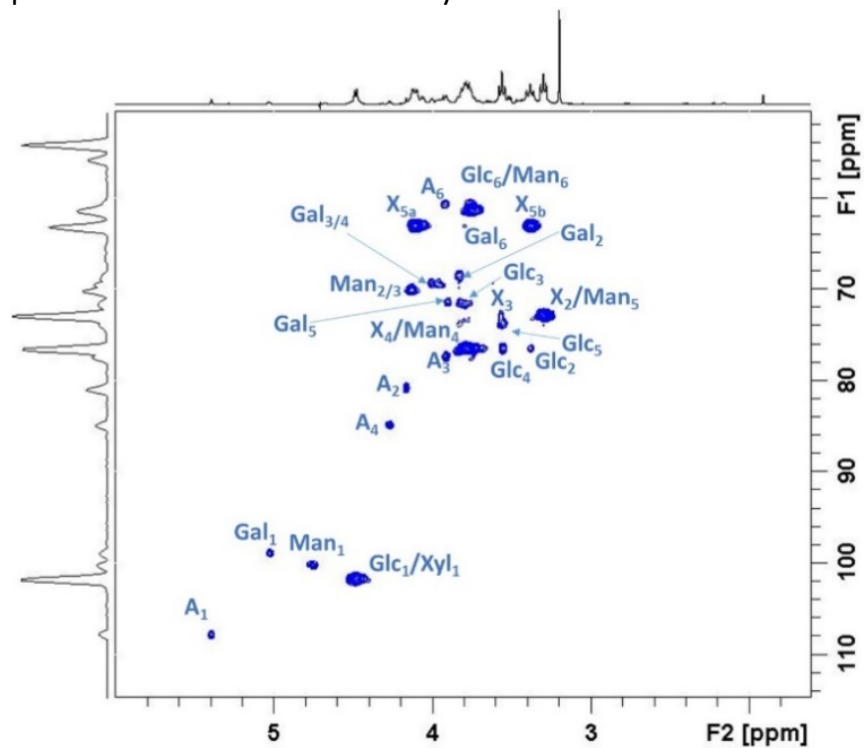

Fig. 6. Hemicellulose fraction from DES treatment after ethanol precipitation, drying and $\mathrm{D}_{2} \mathrm{O}$ dissolution.

\section{DES cost estimation and recycle}

The sustainability of this process is related to, at least, two key factors: the DESs cost and their recyclability. Unlike common choline chloride-based DESs, the cost of DESs studied in the present work is strongly influenced by the high cost of choline acetate $(116$ euro $/ \mathrm{kg}$ ) which compares unfavourably with cheap ChCl (1-1.2 euro/kg). The costs of $\mathrm{ChCl}$ - and ChOAcbased DESs, estimated using the best quotation of raw materials (Table S3+) and the following equation 3 are reported in Table 5 ,

$$
\text { DES Price }=\frac{M_{H B A} P_{H B A}+M_{H B D} P_{H B D}}{M_{H B A}+M_{H B D}} \text { [eqn 3] }
$$

where $M_{H B A}$ and $M_{H B D}$ are the molecular weights of the $H$ bonds acceptor (HBA) and $\mathrm{H}$-bonds donor (HBD), while $P_{H B A}$ and $P_{H B D}$ are their prices. The cost of ChOAc-based DESs turns out to be 24 times higher than the analogous $\mathrm{ChCl}$-based DESs when glycolic acid is used as HBD and up to 50 times when imidazole is futures as HBD. However, it is worth mentioning that $\mathrm{ChCl}$ is widely used as a feed additive, while choline acetate is not currently employed in any large-scale application. This aspect is a relevant one for any low-volume production goods and likely lies beneath the big difference between the costs of the two products. The bulk cost of ChOAc (from now on $\mathrm{ChOAc}_{\mathrm{opt}}$ ) can be reasonably assessed applying the equation reported by Hallett et al. ${ }^{87}$ for the synthesis of protic ionic liquids. In their work Hallett et al. concluded that, for syntheses that require a few steps, the cost of the products is largely influenced by the cost of raw materials while fixed and operational costs account for a maximum of $25 \%$ of raw material. For the synthesis of $\mathrm{ChOAc}_{\mathrm{opt}}$, it can be reasonably assumed that the cost of the product can be estimated by using a similar equation. For a conservative estimation of the final cost, a value of $45 \%$ of raw material cost was allocated to fixed and operational costs. Taking into account these aspects, a new equation for cost calculation for $\mathrm{ChOAc}_{\mathrm{opt}}$ production was developed.

$$
\mathrm{ChOAc}_{\mathrm{opt}}=\left(\frac{M_{C h C l} P_{C h C l}+M_{A A} M_{A A}}{M_{C h C l}+M_{A A}}\right) 1.45 \quad \text { [eqn 4] }
$$

In equation $4, \mathrm{M}_{\mathrm{ChCl}}$ and $\mathrm{M}_{\mathrm{AA}}$ are the molecular weights of choline chloride $(\mathrm{ChCl})$ and acetic acids $(\mathrm{AA})$ while $\mathrm{P}_{\mathrm{ChCl}}$ and $\mathrm{P}_{\mathrm{AA}}$ are their prices. Therefore, applying equation 4 and using the best quotation of choline chloride and acetic acid (listed in the supporting information), it is possible to estimate the cost of $\mathrm{ChOAc}_{\mathrm{opt}}$ at about 1.5 euro/kg. Starting from this value and using equation 3 , the cost of $\mathrm{ChOAc}_{\text {opt }}$-based DESs was computed and is shown in Table 5. 
Table 5. Estimated cost of ChOAc- and ChCl-based DES

\section{ChOAC:HBD 1:1 ChCl:HBD 1:1 ChOAC $\quad$ HBD 1:1}

\begin{tabular}{cccccccccc}
\hline $\begin{array}{c}\text { HBD } \\
\begin{array}{c}\text { Cost } \\
\text { (euro/kg) }\end{array}\end{array}$ & Glx & Im & LevA & Glx & Im & LevA & Glx & Im & LevA \\
\hline
\end{tabular}

Therefore, the final costs of $\mathrm{ChOAc}_{\mathrm{opt}}$-based DESs are very similar to their $\mathrm{ChCl}$ analogues.

The recycle of DES is the other key variable for the economic sustainability of the process, as well as the quality of the cellulose obtained by the process using the recovered solvent. In the present section we report, as a paradigmatic example, the tests carried out on ChOAc:LevA. As shown in ${ }^{1} \mathrm{H}$ NMR spectra (Fig. S40 ${ }^{\dagger}$ ), there are no differences between the fresh and the recycled DESs. Throughout the four cycles, ChOAc:LevA maintained its chemical structure and its capability to purify the Kraft cellulose by selective solubilisation of hemicellulose and lignin. In Fig. 7 the performance of ChOAc:LevA is reported for the first 4 cycles (green bars of histogram). The recovered yield of the DES solution was evaluated by mass measurement and it resulted to be about $90 \%$ per cycle.

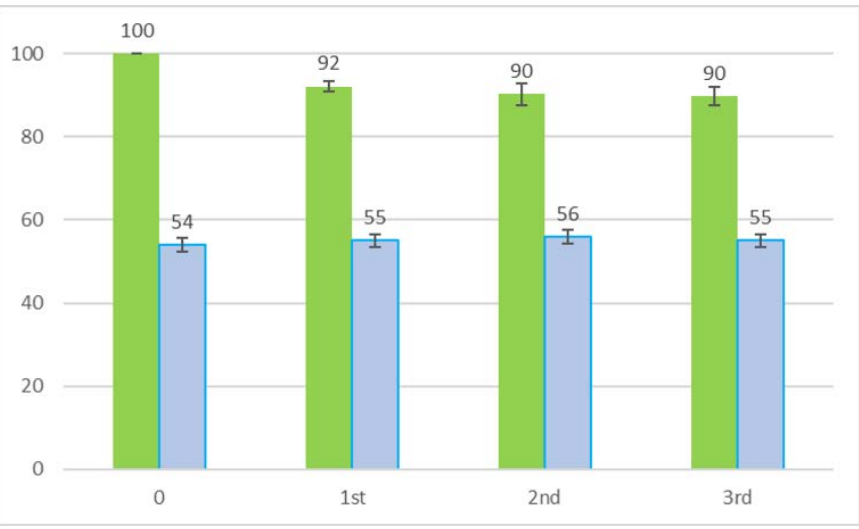

Fig. 7. Recovery yield (\%) of ChOAc:LevA for the four consecutive cycles of Kraft cellulose treatment (green bars) and crystallinity index of cellulose (\%) after the DES treatment (blue bars).

The blue bars of the histogram of Fig. 7 show the measured crystallinity index of the cellulose after each cycle. It is clear that the crystallinity index of the purified cellulose is also constant throughout the considered cycles.

\section{Experimental}

\section{Chemicals}

Choline acetate (98\%) was purchased from lolitech. Glycolic acid (99\%), Levulinic acid (99\%), Imidazole (99\%) and microcrystalline cellulose were purchased from Sigma-Aldrich. Xylan from beechwood was purchased from Serva. Kraft cellulose obtained from Kraft process was provided by Akzo Nobel Chemicals S.p.A.

\section{DESs preparation}

DESs were prepared according to one of the most used procedure reported in the literature. Briefly, the preparation involved the combination of choline acetate (HBA) with three different HBDs, glycolic acid, levulinic acid and imidazole at $80^{\circ} \mathrm{C}$ for $30 \mathrm{~min}$ stirring, until a homogeneous and transparent solution was formed. The prepared DESs were cooled and used for our solubility tests without any purification.

\section{NMR Spectroscopy}

For the NMR experiments $20 \mathrm{mg}$ of sample was dissolved in $0.60 \mathrm{~mL}$ of $\mathrm{D}_{2} \mathrm{O}$ (99.97\%) containing TSP (trimethylsylilpropionic acid) as internal chemical shift reference.

${ }^{1} \mathrm{H}$ NMR experiments were carried out on a Bruker NEO 500 spectrometer equipped with a $5 \mathrm{~mm}$ pulsed-field z-gradient BBFO probe. For each sample, the probe was carefully tuned, and the $90^{\circ}$ pulses evaluated. All NMR spectra were recorded at $298 \mathrm{~K}$ : the sample temperature was set and controlled using a variable temperature control unit. Water suppression was achieved by low-power presaturation of the residual HOD signal.

HSQC and TOCSY experiments were recorded for the assignment of reference and Kraft hemicellulose. HSQC experiments were recorded using a pulse program with water presaturation (hsqcetgpsiwt) and a data matrices of 2048 (t2) $x$ 256 (t1) complex data points. 32 transients were accumulated per increment. TOCSY spectra were recorded with a $\tau_{\text {mix }}$ of 0.08 s using data matrices of 2048 (t2) x 256 (t1) complex data points. 16 transients were accumulated per increment. Raw data were processed by applying a cosine squared sine window functions in both dimensions. All data were processed using the Bruker's Topspin 4.0.3 software.

\section{XRD}

The experiments were performed with a Bruker D2 Phaser Xray powder diffractometer using CuKa radiation. The data were collected in the $2 \theta$ range $7^{\circ}-40^{\circ}$ with a step size of $0.02^{\circ}$ and a counting time of $0.4 \mathrm{~s}$ per step, a primary slit module of $0.6 \mathrm{~mm}$, air scatter screen module $1 \mathrm{~mm}$ and secondary slit module of $8 \mathrm{~mm}$.

\section{Viscosity}

\section{Viscosity $(\eta)$ was determined using Brookfield DV-II + Pro} (Brookfield AMETEK, Inc., Middleboro, MA, USA) programmable viscometer, with an uncertainty of $\pm 2 \%$ in the temperature range from 20 to $90{ }^{\circ} \mathrm{C}$ (controlled by a Brookfield TC-502 thermostat with an accuracy of $\pm 0.1^{\circ} \mathrm{C}$ ). Viscosity $(\eta)$ was determined in the temperature range from 20 to $90{ }^{\circ} \mathrm{C}$, 
applying 15 different shearing rates between $1 \mathrm{~s}^{-1}$ and $100 \mathrm{~s}^{-1}$. The resulting average values are given in SI Table 1 and Fig. S3. Since DESs are very hygroscopic substances, every sample was heated at $35^{\circ} \mathrm{C}$ under high vacuum for $45 \mathrm{~min}$ before each measurement to eliminate the effect of water. The water content of DESs was estimated by Karl Fischer titration using a SI Analytics coulometer (Titroline 75000 KFtrace) and the water content was 183 ppm for ChOAc:LevA, 201 ppm ChOAc:GlyA, and 168 ppm for ChOAc:Im.

\section{DSC}

DSC analyses were performed with 5-10 $\mathrm{mg}$ of sample in pin hole aluminium pan at different scanning rate $\left(2-10{ }^{\circ} \mathrm{C} / \mathrm{min}\right)$ in nitrogen flow in the temperature range going from $-90{ }^{\circ} \mathrm{C}$ to $100{ }^{\circ} \mathrm{C}$. In the drying cycle, the sample was heated from $40^{\circ} \mathrm{C}$ to $100^{\circ} \mathrm{C}$ and maintained at this temperature for $30 \mathrm{~min}$. Then, it was cooled down from $100{ }^{\circ} \mathrm{C}$ to $-90^{\circ} \mathrm{C}$, at a rate of 10 ${ }^{\circ} \mathrm{C} / \mathrm{min}$, and maintained at $-90^{\circ} \mathrm{C}$ for 5 minutes (cooling run). Finally, the sample was heated to $100^{\circ} \mathrm{C}$ at a rate of $10^{\circ} \mathrm{C} / \mathrm{min}$ (heating run). Three different heating rates were performed 10 ${ }^{\circ} \mathrm{C} / \mathrm{min}, 5^{\circ} \mathrm{C} / \mathrm{min}$, and $2{ }^{\circ} \mathrm{C} / \mathrm{min}$. DSC experiments were carried out in duplicate.

\section{TGA}

The thermal stability of prepared DESs, hemicellulose and cellulose was investigated by thermal gravimetric analysis (TGA), conducted in a TA Instruments Q500 TGA. The sample (15-20 mg) was heated in a platinum crucible as sample holders. First, the heating mode was set to isothermal at $50^{\circ} \mathrm{C}$ in $\mathrm{N}_{2}(100 \mathrm{~mL} / \mathrm{min})$ for $30 \mathrm{~min}$. Then, the sample was heated from 30 to $700{ }^{\circ} \mathrm{C}$ with a heating rate of $10^{\circ} \mathrm{C} / \mathrm{min}$ under nitrogen $(100 \mathrm{~mL} / \mathrm{min})$. Mass change was recorded as a function of temperature and time. TGA experiments were carried out in duplicate.

\section{FT-IR}

FT-IR spectra were recorded using a Varian 660 spectrometer. The transmission technique with the $\mathrm{KBr}$ pellet was chosen. Spectra were recorded using 64 scans in the range of $600-4000$ $\mathrm{cm}^{-1}$ with a spectra resolution of $4 \mathrm{~cm}^{-1}$. Pellets were prepared by weighing $300 \mathrm{mg}$ of $\mathrm{KBr}$ and $3 \mathrm{mg}$ of sample.

\section{Kraft Cellulose DES treatment}

$10 \mathrm{~g}$ of DESs were prepared and the $10 \mathrm{wt}$. \% of Kraft cellulose (1g) was added, then the mixture was left stirring at $80^{\circ} \mathrm{C}$ for $24 \mathrm{~h}$. After the treatment, the DESs/cellulose mixtures changed their aspect into a homogeneous gel. Cellulose was selectively precipitated by adding an excess of water to the mixture at the same temperature. The regenerated cellulose thus obtained was washed with $10 \mathrm{~mL}$ of water, centrifuged 4 times to remove all DES residues and provide suitable samples for the analysis.

Ethanol was then added to the supernatant in order to precipitate hemicellulose fraction. The precipitate was washed 6 times with $10 \mathrm{~mL}$ of ethanol, dried and re-dissolved in deuterium oxide for NMR analysis.

\section{Kraft cellulose $\mathrm{NaOH}$ treatment}

$200 \mathrm{mg}$ of cellulose was stirred in $4 \mathrm{~mL}$ of $\mathrm{NaOH} 18 \%$ solution for $24 \mathrm{~h}$. The solid residue was filtered, the solution was neutralized using the cation exchange resin Amberlite IR-120. The sample was dried and re-dissolved in deuterium oxide for NMR analysis.

\section{Holocellulose content}

Based upon the procedures described by Yokoyama et al. (2002).$^{88}$ In a round bottom flask, $1 \mathrm{~g}$ of dried kraft cellulose in $20 \mathrm{~mL}$ water was heated with stirring to $90^{\circ} \mathrm{C}$. A sodium chlorite solution ( $20 \mathrm{wt} . \%, 5 \mathrm{~mL}$ ) and $2 \mathrm{~mL}$ of glacial acetic acid were then added. The addition of sodium chlorite solution and glacial acetic acid was repeated at 30,60 and 90 min after the first addition. $2 \mathrm{~h}$ after the first addition, the round bottom flask was cooled in a cold-water bath. Glass microfibers filter was used to filter the reaction mixture. The residue, holocellulose, was washed with hot water $(3 \times 200 \mathrm{~mL})$ and acetone $(20 \mathrm{~mL})$ and dried at $105^{\circ} \mathrm{C}$. The holocellulose content was quoted as wt. \% of the dried Kraft cellulose weight.

\section{Alpha cellulose and hemicellulose content R18 (method used for} cellulose pulps)

For bleached and delignified pulp, the level of cellulose purity is obtained by R10, R18, S10 and S18 methods. ${ }^{89}$ The values represent the pulp's solubility in $10 \%$ and $18 \% \mathrm{NaOH}$ solutions under specified conditions (standard ISO 692:1982). The soluble portion (\%) of pulp in $10 \%$ and $18 \% \mathrm{NaOH}$ is referred to as S10 and S18, respectively, while the residual fraction (\%) is referred to as R10 and R18. It is known that a $10 \% \mathrm{NaOH}$ solution can dissolve the degraded cellulose and hemicelluloses (S10), whereas an $18 \% \mathrm{NaOH}$ solution dissolves the most important part of hemicelluloses (S18). The subtraction, S10-S18 (or R18-R10), is a measure of the degraded cellulose, S18 (or 100-R18) represents hemicelluloses and R10 corresponds to $\alpha$-cellulose and represents the "long-chain" cellulose content.

$500 \mathrm{mg}$ of the holocellulose obtained from the above reaction was placed in $100 \mathrm{~mL}$ beaker and left for $30 \mathrm{~min}$ to allow moisture equilibrium. $40 \mathrm{~mL} \mathrm{NaOH}(10 \% \mathrm{w} / \mathrm{v})$ was added and left for $30 \mathrm{~min} .40 \mathrm{~mL}$ of water was added and stirred for $1 \mathrm{~min}$ with a glass stirring rod then left for another $29 \mathrm{~min}$. The suspension was filtered with a sintered glass filter and washed with deionised water $(3 \times 100 \mathrm{~mL})$. The residue, was soaked in $1 \mathrm{M}$ acetic acid $(10 \mathrm{~mL}$ ) for $5 \mathrm{~min}$. It was then filtered and 
washed with deionised hot water $(3 \times 300 \mathrm{~mL})$ followed by drying at $105^{\circ} \mathrm{C}$. The same procedure was repeated using $\mathrm{NaOH} 18 \% \mathrm{w} / \mathrm{v}$.

\section{Fock's reactivity measurements}

$500 \mathrm{mg}$ of dry cellulose was weighed and placed in a $250 \mathrm{~mL}$ flask with a stopper. $50 \mathrm{~mL}$ of $\mathrm{NaOH} 9 \mathrm{wt} . \%$ solution was added, and shaken in a water bath for $10 \mathrm{~min}$ at $19^{\circ} \mathrm{C}$. The flask was sealed with the stopper as soon as $1.3 \mathrm{~mL} \mathrm{CS} \mathrm{CS}_{2}$ was added and the mixture was stirred for 3 hours. At the end distilled water was added to the flask to give the solution a total mass of $100 \mathrm{~g}$. The solution was centrifuged at $4000 \mathrm{rpm}$ for $20 \mathrm{~min}$. $10 \mathrm{~mL}$ of the supernatant was pipetted into a $100 \mathrm{~mL}$ flask, neutralized with $3 \mathrm{~mL}$ sulfuric acid $20 \mathrm{wt} . \%$, left for $15 \mathrm{~h}$, to obtain the regenerated cellulose. To determine the dissolved cellulose, $20 \mathrm{~mL}$ of sulfuric acid $68 \mathrm{wt} . \%$ was added to the $100 \mathrm{~mL}$ flask, and the suspension was shaken for $1 \mathrm{~h}$ to acidify the regenerated cellulose. $10 \mathrm{~mL}$ of potassium dichromate solution (1/6 M) was added to the flask, and the mixture was boiled for $1 \mathrm{~h}$ to complete oxidation. The cold mixture was diluted to $100 \mathrm{~mL}$. $40 \mathrm{~mL}$ of this solution was transferred into a $250 \mathrm{~mL}$ flask and titrated with sodium thiosulfate $(0.1 \mathrm{~N})$ using starch as the indicator after $5 \mathrm{~mL}$ potassium iodide $(10 \% \mathrm{w} / \mathrm{w})$ was added. The volume of consumed sodium thiosulfate was recorded to calculate the percentage of cellulose that reacted with $\mathrm{CS}_{2}$.

\section{Conclusions}

In conclusion, the results show that the novel DESs presented in this work are very efficient and promising media for cellulose purification from lignin and hemicellulose. In particular, ChOAc:LevA and ChOAc:Im showed a great efficiency in the solubilisation of hemicellulose from Kraft cellulose samples, thus providing clear evidence of cellulose/hemicellulose/lignin separation. These achievements are due to simple DES solubilisation of the non-crystalline components and their re-precipitation by means of environmentally mild anti-solvents (such as ethanol and water). This protocol offers a great potential in the field of biomass valorization and its appeal is increased by the easy recovery of hemicellulose. The case-study of the Kraft cellulose purification described in the final part of this work shows how the DES process is a valid, mild and non- destructive alternative to the current alkali-based technology. It is worth stressing that the DES systems described in this work showed different capability of dissolving lignin. These findings further confirm the tunability of DESs properties by changing the HBD donors and pave the way for further subtle lignocellulosic biomass fractionation challenges. Finally, the benign effect of water, up to $15 \mathrm{wt}$. \%, in the hemicellulose solubilisation adds value to the process in view of scaling up towards industrial applications. Indeed, the presence of water decreases the viscosity, reduces the amount of DES required for the dissolution process and saves costs in DES recycling, since an energy-intensive drying step would be avoided.

\section{Conflicts of interest}

There are no conflicts to declare.

\section{Acknowledgements}

GCD thanks Politecnico di Milano for the Interdepartmental PhD Programme scholarship.

\section{References}

D. Klemm, B. Philipp, T. Heinze, U. Heinze and W. Wagenknecht, in Comprehensive Cellulose Chemistry, 2004, vol. 2, pp. 1-31.

A. Guleria, G. Kumari and S. Saravanamurugan, in Biomass, Biofuels, Biochemicals, Elsevier B.V., 2020, pp. 433-457. D. L. Kaplan, ed. D. L. Kaplan, Springer Berlin Heidelberg, Berlin, Heidelberg, 1998, pp. 1-29.

4 T. Heinze, O. A. El Seoud and A. Koschella, eds. T. Heinze, O. A. El Seoud and A. Koschella, Springer International Publishing, Cham, 2018, pp. 39-172.

5 H. Sixta, Handbook of Paper and Board, 2006.

6 F. Haemmerle, in Lenzinger - berichte, 2011, pp. 12-21.

7 Risi Fastmarkets, Outlook for the Global Dissolving Pulp Market, 2019.

8 International Cotton Advisory Committee.

9 H. Sixta, M. lakovlev, L. Testova, A. Roselli, M. Hummel, M. Borrega, A. van Heiningen, C. Froschauer and $\mathrm{H}$. Schottenberger, Cellulose, 2013, 20, 1547-1561.

10 Y. Shi, T. Akiyama, M. Yashiro and Y. Matsumoto, BioResources, 2013, 8, 4837-4848.

11 J. Li, Y. Liu, C. Duan, H. Zhang and Y. Ni, Bioresour. Technol., 2015, 192, 501-506.

12 V. B. Agbor, N. Cicek, R. Sparling, A. Berlin and D. B. Levin, Biotechnol. Adv., 2011, 29, 675-685. J. P. Delgenes, V. Penaud and R. Moletta, Cheminform, DOI:10.1002/chin.200313271. V. L. Budarin, J. H. Clark, B. A. Lanigan, P. Shuttleworth and D. J. Macquarrie, Bioresour. Technol., 2010, 101, 37763779.

X. Zhao, Y. Zhou, G. Zheng and D. Liu, Appl. Biochem. Biotechnol., 2010, 160, 1557-1571.

16 A. García, M. G. Alriols, R. Llano-Ponte and J. Labidi, Bioresour. Technol., 2011, 102, 6326-6330. H. A. Villa-Vélez, H. A. Váquiro and J. Telis-Romero, Ind. Crops Prod., 2015, 66, 52-61. B. Deepa, E. Abraham, B. M. Cherian, A. Bismarck, J. J. Blaker, L. A. Pothan, A. L. Leao, S. F. de Souza and M. Kottaisamy, Bioresour. Technol., 2011, 102, 1988-1997. A. Romaní, G. Garrote, I. Ballesteros and M. Ballesteros, Fuel, 2013, 111, 66-74.

A. Romaní, H. A. Ruiz, F. B. Pereira, L. Domingues and J. A Teixeira, Ind. Eng. Chem. Res., 2013, 52, 14342-14352. Y. Sun and J. Cheng, 2002, 83, 1-11. 
673-686.

23

E. Ruiz, I. Romero, M. Moya, C. Cara, J. D. Vidal and E.

Castro, Bioresour. Technol., 2013, 140, 292-298.

24

Y. Chen, M. A. Stevens, Y. Zhu, J. Holmes and H. Xu,

Biotechnol. Biofuels, 2013, 6, 8.

R. Travaini, M. D. M. Otero, M. Coca, R. Da-Silva and S. Bolado, Bioresour. Technol., 2013, 133, 332-339.

M. G. Alriols, A. Tejado, M. Blanco, I. Mondragon and J.

Labidi, Chem. Eng. J., 2009, 148, 106-114.

S. Singh, G. Cheng, N. Sathitsuksanoh, D. Wu, P. Varanasi,

A. George, V. Balan, X. Gao, R. Kumar, B. E. Dale, C. E.

Wyman and B. A. Simmons, Front. Energy Res., 2015, 3, 62.

D. A. Fort, R. C. Remsing, R. P. Swatloski, P. Moyna, G.

Moyna and R. D. Rogers, Green Chem., 2007, 9, 63-69.

. H. Lee, T. V Doherty, R. J. Linhardt and J. S. Dordick,

Biotechnol. Bioeng., 2009, 102, 1368-1376.

30

S. K. Karatzos, L. A. Edye and W. O. S. Doherty, Biotechnol.

Biofuels, 2012, 5, 1-12.

31 D. Fu, G. Mazza and Y. Tamaki, J. Agric. Food Chem., 2010, 58, 2915-2922.

32 K. M. Torr, K. T. Love, B. A. Simmons and S. J. Hill, Biotechnol. Bioeng., 2016, 113, 540-549.

T. Dutta, J. Shi, J. Sun, X. Zhang, G. Cheng, B. A. Simmons, S. Singh, M. M. Pereira, J. A. P. Coutinho and M. G. Freire, Ionic Liquids in the Biorefinery Concept, The Royal Society of Chemistry, 2015, vol. 2016- Janua.

A. Pinkert, K. N. Marsh, S. Pang and M. P. Staiger, Chem. Rev., 2009, 109, 6712-6728.

R. P. Swatloski, S. K. Spear, J. D. Holbrey and R. D. Rogers, J. Am. Chem. Soc., 2002, 124, 4974-4975.

37, 123-150

G. Durga and A. Mishra, in Encyclopedia of Inorganic and Bioinorganic Chemistry, 2016, pp. 1-13.

C. G. Yoo, Y. Pu and A. J. Ragauskas, Curr. Opin. Green Sustain. Chem., 2017, 5, 5-11.

A. M. Stepan, A. Michud, S. Hellstén, M. Hummel and H. Sixta, Ind. Eng. Chem. Res., 2016, 55, 8225-8233.

P. Verdía, A. Brandt, J. P. Hallett, M. J. Ray and T. Welton, Green Chem., 2014, 16, 1617-1627.

A. Roselli, S. Asikainen, A. Stepan, A. Monshizadeh, N. Von Weymarn, K. Kovasin, Y. Wang, H. Xiong, O. Turunen, M. Hummel and H. Sixta, Holzforschung, 2016, 70, 291-296. K. D. O. Vigier, G. Chatel and F. Jérôme, ChemCatChem, 2015, 7, 1250-1260.

E. L. Smith, A. P. Abbott and K. S. Ryder, Chem. Rev., 2014, 114, 11060-11082.

Y. L. Loow, T. Y. Wu, G. H. Yang, L. Y. Ang, E. K. New, L. F. Siow, J. Md. Jahim, A. W. Mohammad and W. H. Teoh, Bioresour. Technol., 2018, 249, 818-825.

M. Zdanowicz, K. Wilpiszewska and T. Spychaj, Carbohydr. Polym., 2018, 200, 361-380.

D. J. G. P. Van Osch, L. J. B. M. Kollau, A. Van Den Bruinhorst, S. Asikainen, M. A. A. Rocha and M. C. Kroon, Phys. Chem. Chem. Phys., 2017, 19, 2636-2665.

Y. P. Mbous, M. Hayyan, A. Hayyan, W. F. Wong, M. A. Hashim and C. Y. Looi, Biotechnol. Adv., 2017, 35, 105-134. R. A. Sheldon, Chem. - A Eur. J., 2016, 22, 12984-12999.

M. Pan, G. Zhao, C. Ding, B. Wu, Z. Lian and H. Lian, Carbohydr. Polym., 2017, 176, 307-314.

Y. L. Loow, E. K. New, G. H. Yang, L. Y. Ang, L. Y. W. Foo and T. Y. Wu, Cellulose, 2017, 24, 3591-3618.
A. Satlewal, R. Agrawal, S. Bhagia, J. Sangoro and A. J. Ragauskas, Biotechnol. Adv., 2018, 36, 2032-2050. B. Tang, H. Zhang and K. H. Row, J. Sep. Sci., 2015, 38, 1053-1064.

C. W. Zhang, S. Q. Xia and P. S. Ma, Bioresour. Technol., 2016, 219, 1-5.

H. Ren, C. Chen, S. Guo, D. Zhao and Q. Wang, BioResources, 2016, 11, 8457-8469.

E. S. Morais, P. V. Mendonça, J. F. J. Coelho, M. G. Freire, C. S. R. Freire, J. A. P. Coutinho and A. J. D. Silvestre, ChemSusChem, 2018, 11, 753-762. K. Ninomiya, T. Yamauchi, C. Ogino, N. Shimizu and K. Takahashi, Biochem. Eng. J., 2014, 90, 90-95.

Q. Zhang, M. Benoit, K. Dea Oliveiraa Vigier, J. Barrault and F. Jérǒme, Chem. - A Eur. J., 2012, 18, 1043-1046.

F. Cheng, H. Wang, G. Chatel, G. Gurau and R. D. Rogers, Bioresour. Technol., 2014, 164, 394-401.

G. Colombo Dugoni, M. E. Di Pietro, M. Ferro, F. Castiglione, S. Ruellan, T. Moufawad, L. Moura, M. F. Costa Gomes, S. Fourmentin and A. Mele, ACS Sustain. Chem. Eng., 2019, 7, 7277-7285.

A. K. Kumar, B. S. Parikh and M. Pravakar, Environ. Sci. Pollut. Res., 2016, 23, 9265-9275.

Y. Dai, G. J. Witkamp, R. Verpoorte and Y. H. Choi, Food Chem., 2015, 187, 14-19.

C. Ma, A. Laaksonen, C. Liu, X. Lu and X. Ji, Chem. Soc. Rev., 2018, 47, 8685-8720.

N. Rodriguez Rodriguez, A. van den Bruinhorst, L. J. B. M. Kollau, M. C. Kroon and K. Binnemans, ACS Sustain. Chem. Eng., 2019, 7, 11521-11528.

Y. Elhamarnah, M. Nasser, H. Qiblawey, A. Benamor, M. Atilhan and S. Aparicio, J. Mol. Liq., 2019, 277, 932-958. M. Zdanowicz, T. Spychaj and H. Maka, Carbohydr. Polym., 2016, 140, 416-423. C. Florindo, F. S. Oliveira, L. P. N. Rebelo, A. M. Fernandes and I. M. Marrucho, ACS Sustain. Chem. Eng., 2014, 2, 2416-2425.

H. Malaeke, M. R. Housaindokht, H. Monhemi and M. Izadyar, J. Mol. Liq., 2018, 263, 193-199.

C. D'Agostino, L. F. Gladden, M. D. Mantle, A. P. Abbott, E. Ahmed I., A. Y. M. Al-Murshedi and R. C. Harris, Phys. Chem. Chem. Phys., 2015, 17, 15297-15304. V. Alizadeh, F. Malberg, A. A. H. Pádua and B. Kirchner, J. Phys. Chem. B, 2020, 124, 7433-7443. B. Soares, D. J. P. Tavares, J. L. Amaral, A. J. D. Silvestre, C. S. R. Freire and J. A. P. Coutinho, ACS Sustain. Chem. Eng., 2017, 5, 4056-4065. Y. Ni, BioResources, 2016, 11, 5553-5564. T. Hannuksela and C. Hervé du Penhoat, Carbohydr. Res., 2004, 339, 301-312. J. Lundqvist, A. Teleman, L. Junel, G. Zacchi, O. Dahlman, F. Tjerneld and H. Stålbrand, Carbohydr. Polym., 2002, 48, 29-39.

$74 \quad$ X. Zhou, W. Li, R. Mabon and L. J. Broadbelt, Energy Technol., 2017, 5, 52-79. B. Arnoul-jarriault, D. Lachenal, C. Chirat and L. Heux, Ind. Crops Prod., 2015, 65, 565-571. W. Chen, Z. Xue, J. Wang, J. Jiang, X. Zhao and T. Mu, Wuli Huaxue Xuebao/ Acta Phys. - Chim. Sin., 2018, 34, 904-911. J. González-Rivera, E. Husanu, A. Mero, C. Ferrari, C. Duce, M. R. Tinè, F. D’andrea, C. S. Pomelli and L. Guazzelli, J. 
Mol. Liq., 2019, 10.1016/j.molliq.2019.112357.

H. Xu, X. Che, Y. Ding, Y. Kong, B. Li and W. Tian, Bioresour. Technol., 2019, 279, 271-280.

P. Ahvenainen, I. Kontro and K. Svedström, Cellulose, 2016, 23, 1073-1086.

80 A. M. Raspolli Galletti, A. D’Alessio, D. Licursi, C. Antonetti, G. Valentini, A. Galia and N. Nassi o Di Nasso, J. Spectrosc., 2015, 2015, 719042.

81 S. Zhou, Y. Li, L. Huang, L. Chen and Q. Miao, BioResources, 2018, 13, 2861-2870.

82 D. Ibarra, V. Köpcke, P. T. Larsson, A. S. Jääskeläinen and M. Ek, Bioresour. Technol., 2010, 101, 7416-7423. W. Fock, Papier, 1959, 13, 92-95.

C. Tian, L. Zheng, Q. Miao, C. Nash, C. Cao and Y. Ni, Tappi J, 2013, 12, 21-26.

85 I. Carrillo-Varela, R. Retamal, M. Pereira and R. T. Mendonça, Cellulose, 2019, 26, 5731-5744.

86 P. Strunk, T. Ö. Öman, M. Hedenström, B. Eliasson and A. Gorzsás, Nord. Pulp Pap. Res. J., 26, 398-409.

87 L. Chen, M. Sharifzadeh, N. Mac Dowell, T. Welton, N. Shah and J. P. Hallett, Green Chem., 2014, 16, 3098-3106.

88 T. Yokoyama, J. F. Kadla and H. Chang, J. Agric. Food Chem., 2002, 50, 1040-1044.

89 M. Carrier, A. Loppinet-Serani, D. Denux, J. M. Lasnier, F. Ham-Pichavant, F. Cansell and C. Aymonier, Biomass and Bioenergy, 2011, 35, 298-307. 